IFUP-TH 42/96

\title{
Classical improvement of lattice actions and quantum effects: a unified view.
}

\author{
Paolo Rossi and Ettore Vicari \\ Dipartimento di Fisica dell'Università and I.N.F.N., I-56126 Pisa, Italy
}

\begin{abstract}
The possibility of removing the one-loop perturbative effects of lattice artifacts by a proper choice of the lattice action is explored, and found to depend crucially on the properties of the physical quantity considered. In this respect the finite-space-volume mass gap $m(L)$ is an improved observable. We find an explicit momentum space representation of the one-loop contribution to $m(L)$ for arbitrary lattice actions in the case of two-dimensional $O(N) \sigma$ models. We define a "tree perfect" Symanzik action and find that it formally removes all one-loop lattice artifacts in $m(L)$. On-shell improved actions do not share this property.
\end{abstract}

PACS numbers: 11.15.Ha 
Improvement of the lattice action has been often advocated as a possible way out of the problem of finite lattice spacing effects, that obscure the scaling properties in numerical simulations on small lattices. The original idea can be traced back to Wilson [1], who introduced the notion of renormalization group trajectory. Lattice actions determined by the trajectory of a renormalization group transformation are free of lattice artifacts. Due to the difficulty of effectively finding a renormalized trajectory, two major strategies of improvement were suggested by Symanzik [2] and by Hasenfratz and Niedermayer [3]. They differ both in purpose and in many technical details, but they share the possibility of defining a "classically perfect action" whose properties may then be compared. By "classically perfect action" we mean a lattice action which does not present any power-like dependence on the lattice spacing in the tree evaluation of spectral properties, and of properly chosen correlation functions.

In Symanzik's case, this action is of no practical relevance, since it involves infinitelylong-range interactions. However its properties are theoretically interesting in view of a deeper understanding of the whole improvement program.

By renormalization group arguments, one can infer that quantum effects are more important than higher order classical finite lattice spacing contributions. It is therefore worth exploring the mechanism that might lead to important cancellations of these quantum effects. Symanzik's "tree perfect" action constitutes a reasonably simple laboratory in order to check some ideas that have been put forward.

Hasenfratz and collaborators [4] stressed that in two-dimensional $O(N) \sigma$ models their version of a "classically perfect" action showed no power-like dependence on the lattice artifacts in the one-loop contribution to the finite-space-volume mass gap. Such lattice artifacts are instead found when calculating the same quantity with the standard nearestneighbor action. The same authors already observed that the above effects were consistently reduced in the simplest (second-nearest-neighbor) version of Symanzik improvement. Their approach was however intrinsically numerical, and therefore not easily adapted to exploring the different possibilities in full generality. We therefore decided to move a step forward, and to look for an analytic expression of the one-loop contribution to the finite-space-volume mass gap in two-dimensional $O(N) \sigma$ models, that could hold for an arbitrary form of the lattice action, allowing a systematic study of finite-lattice effects.

We were greatly helped in our effort by the friendly collaboration of M. Lüscher and P. Weisz, who generously made their private notes available to us.

For definiteness, let us recall that any reasonable definition of finite-space-volume mass gap $m(L)$ ( $L$ is the size of the space volume) in 2-d $O(N) \sigma$ models admits a loop expansion in the form [5] [6]:

$$
m(L) L=\frac{N-1}{2} g^{2} \sum_{l=0}^{\infty} A_{l}(L) g^{2 l}
$$

where $l$ is the number of loops and $A_{l}(L)$ in turn have an asymptotic expansion in powers of $a^{2} / L^{2}$ :

$$
A_{l}(L)=\sum_{n=0}^{\infty}\left(\frac{a^{2}}{L^{2}}\right)^{n} \sum_{p=0}^{l} a_{l p}^{(n)}\left(\ln \frac{L}{a}\right)^{l-p} .
$$


We are neglecting possible exponentially depressed finite-lattice spacing corrections. The coefficients $a_{l p}^{(n)}$ are in general dependent on $N$. Recursive relations between the coefficients $a_{l p}^{(n)}$ ( $n$ fixed and different from zero) are dictated by the renormalization group properties of the higher dimensional operators, whose effective presence in the lattice action generates the scaling violations to $O\left[\left(a^{2} / L^{2}\right)^{n}\right]$ [7]. Terms with $n=0$, i.e. the limit $a^{2} / L^{2} \rightarrow 0$ of $m(L) L$, define a running coupling constant $\bar{g}$ [6],

$$
\bar{g}^{2}=g^{2} \sum_{l=0}^{\infty} \sum_{p=0}^{l} g^{2 l} a_{l p}^{(0)}\left(\ln \frac{L}{a}\right)^{l-p},
$$

obeying a homogeneous renormalization group equation.

It is convenient to reexpress the general result into the form

$$
m(L) L=\frac{N-1}{2} g^{2} \sum_{n=0}^{\infty}\left(\frac{a^{2}}{L^{2}}\right)^{n} \sum_{p=0}^{\infty} g^{2 p} b_{p}^{(n)}\left(g^{2} \ln L / a\right)
$$

where

$$
b_{p}^{(n)}(x)=\sum_{q=0}^{\infty} a_{p+q, p}^{(n)} x^{q}
$$

are completely determined by $a_{p p}^{(n)}$ and the RG recursion equations.

Symanzik's improvement program may act separately on the indices $p$ and $n$, which are respectively related to higher-loop contributions and to insertions of higher-dimensional operators. In the usual language, tree-improvement would consist in removing the coefficients $a_{00}^{(n)}$ for $n \neq 0$, which automatically would also imply removing all coefficients $a_{q 0}^{(n)}$, i.e. setting $b_{0}^{(n)}=0$. In turn, one-loop improvement would amount to setting $a_{11}^{(n)}=0$, that is $b_{1}^{(n)}=0$. However, this point of view would be misleading for the problem at hand. The basic reason for this statement stays in fact that the condition $a_{00}^{(n)}=0$ for $n \neq 0$ (i.e. $A_{0}(L)=1$ ) is obtained automatically, for any action, by a proper choice of the definition of $m(L)$, making no reference whatsoever to any improvement program.

The finite-space-volume mass gap $m(L)$ can be defined as the coefficient of the exponential decay of the wall-wall correlation function in the time direction, i.e. for $\left|x_{0}-y_{0}\right| \rightarrow \infty$

$$
\frac{1}{L^{2}} \sum_{x_{1}, y_{1}}\left\langle s_{x} \cdot s_{y}\right\rangle \sim \exp \left[-m(L)\left|x_{0}-y_{0}\right|\right] .
$$

Exponential decay in perturbation theory is insured by taking free boundary conditions in time, and periodic boundary conditions in space $(L$ points in a circle) [5] [6].

As we shall see, $m(L)$ enjoys the properties that $A_{0}(L)=1$ in the expansion (11) independently of the choice of lattice action. The renormalization group then predicts the one-loop result $a_{10}^{(n)}=0$ for $n \neq 0$, i.e. absence of terms proportional to $\ln (L / a)$ in the finite lattice one-loop corrections to the mass gap.

Which whould be in this case the effect of a tree-improvement, if any? As one might have conjectured Symanzik tree-improvement will now act on the coefficients $a_{11}^{(n)}$, setting it 
to zero. As a consequence we would have $b_{1}^{(n)}=0$ as a tree-improvement effect, without the need to appeal to any notion of one-loop quantum improvement.

In order to produce evidence for these statements, let us consider the explicit expression of the one-loop contributions to $m(L) L$. At the one-loop level, full generality is achieved starting from a lattice action whose parametrization is the following [3]:

$$
\mathcal{A}=-\frac{1}{2} \sum_{n_{1} n_{2}} \rho_{n_{1} n_{2}}\left(1-s_{n_{1}} \cdot s_{n_{2}}\right)+\sum_{n_{1} n_{2} n_{3} n_{4}} c_{n_{1} n_{2} n_{3} n_{4}}\left(1-s_{n_{1}} \cdot s_{n_{2}}\right)\left(1-s_{n_{3}} \cdot s_{n_{4}}\right) .
$$

$n_{i}$ are lattice sites, and the functions $\rho_{n_{1} n_{2}}$ and $c_{n_{1} n_{2} n_{3} n_{4}}$ enjoy the following exchange symmetries

$$
\begin{gathered}
\rho_{n_{1} n_{2}}=\rho_{n_{2} n_{1}}, \\
c_{n_{1} n_{2} n_{3} n_{4}}=c_{n_{2} n_{1} n_{3} n_{4}}=c_{n_{1} n_{2} n_{4} n_{3}}=c_{n_{3} n_{4} n_{1} n_{2}} .
\end{gathered}
$$

Possible higher order interactions in the lattice action do not contribute at one-loop. We shall not assume translation invariance, at least in the time direction, since it is in general violated by arbitrary boundary conditions.

We must now compute the two-point spin-spin correlation function up to $O\left(g^{4}\right)$ without relying on any specific feature of the action and for arbitrary boundary conditions. We follow the coordinate space approach [5] in the version suggested by Cline [8]. We introduce the massless propagator $P_{x y}$ which is a solution of the difference equations

$$
\sum_{z} \rho_{x z} P_{z y}=\delta_{x y}-\frac{1}{V}
$$

where $V$ is the total lattice volume. We have removed the zero-mode by appropriate gaugefixing [9]. $P_{x y}$ will not in general be translation-invariant, as a consequence of the noninvariance of $\rho_{x y}$.

The resulting coordinate-space two-point function is

$$
\begin{aligned}
\left\langle s_{x} \cdot s_{y}\right\rangle= & 1+g^{2}(N-1) G_{x y} \\
& +g^{4}(N-1) \frac{1}{2}\left[G_{x y}^{2}+\sum_{n_{1} n_{2}}\left(\rho_{n_{1} n_{2}} G_{n_{1} n_{2}}-\delta_{n_{1} n_{2}}\right) \Delta G_{x y, n_{1}} \Delta G_{x y, n_{2}}\right] \\
& +g^{4}(N-1)^{2} \frac{1}{4} \sum_{n_{1}}\left(\frac{2}{V}+\sum_{n_{2}} \rho_{n_{1} n_{2}} P_{n_{2} n_{2}}\right)\left(\Delta G_{x y, n_{1}}\right)^{2} \\
& +g^{4}(N-1) 4 \sum_{n_{1} n_{2} n_{3} n_{4}} c_{n_{1} n_{2} n_{3} n_{4}}\left(\Delta G_{x y, n_{1}}-\Delta G_{x y, n_{2}}\right)\left(\Delta G_{x y, n_{3}}-\Delta G_{x y, n_{4}}\right) G_{n_{1} n_{3}} \\
& -g^{4}(N-1)^{2} \sum_{n_{1} n_{2} n_{3} n_{4}} c_{n_{1} n_{2} n_{3} n_{4}}\left(\Delta G_{x y, n_{1}}-\Delta G_{x y, n_{2}}\right)^{2} G_{n_{3} n_{4}},
\end{aligned}
$$

where we have introduced the notations

$$
\begin{aligned}
& G_{x y}=P_{x y}-\frac{1}{2}\left(P_{x x}+P_{y y}\right), \\
& \Delta G_{x y, n}=G_{x n}-G_{y n} .
\end{aligned}
$$


It is worth noticing that in the case of open boundary conditions $G_{x y}$ is time-translation invariant up to terms that are exponentially depressed in the time distance $\left|x_{0}-y_{0}\right|$.

When considering space translation invariance, say by taking periodic boundary conditions in space, one can actually perform a Fourier transform of the space coordinate and reduce the problem to an effective one-dimensional equation in time. In the case of open boundary conditions, only the zero-space-momentum component of the propagator will be seriously affected by the lack of translation invariance; all other components will have violations of invariance that are exponentially depressed with the time distance [6].

In view of the above considerations, one may avoid solving the one-dimensional problem with free boundary conditions for an arbitrary form of the action. Indeed the solution for the most general action (7) turns out to differ from that of the standard (nearest-neighbor) action only by terms that are exponentially depressed in time, and therefore not sensitive to the boundaries. As a consequence we may express the general result for $m(L) L$ in terms of the standard action result plus terms that can be computed with periodic boundary conditions, hence directly in momentum space.

Without belaboring on the details of the derivations, we may express our final result for $m(L)$ in terms of the following Fourier transforms:

$$
\begin{gathered}
\widetilde{\rho}(p)=\frac{1}{V} \sum_{n_{1} n_{2}} \rho_{n_{1} n_{2}} e^{-i p \cdot\left(n_{1}-n_{2}\right)}, \\
\widetilde{c}(p, q, r)=\frac{1}{V^{3}} \sum_{n_{1} n_{2} n_{3} n_{4}} c_{n_{1} n_{2} n_{3} n_{4}} e^{-i p \cdot\left(n_{1}-n_{2}\right)} e^{-i q \cdot\left(n_{3}-n_{4}\right)} e^{-i r \cdot \frac{1}{2}\left(n_{1}+n_{2}-n_{3}-n_{4}\right)},
\end{gathered}
$$

where complete symmetrization of the dummy indices, space periodicity and the limit $T \rightarrow \infty$ are assumed. Notice that $\widetilde{c}(p, q, r)$ enjoys also the following symmetry $\widetilde{c}(p, q, r)=$ $\widetilde{c}(q, p, r)$. We introduce the parametrization

$$
A_{1}(L)=r_{1}(L)+(N-2) r_{2}(L)+s_{1}(L)+(N-1) s_{2}(L)
$$

where $s_{i}$ are vanishing when $c_{n_{1} n_{2} n_{3} n_{4}}=0$. We obtained

$$
\begin{gathered}
r_{1}(L)=-\frac{1}{L} \sum_{l=1}^{L} \int \frac{d k_{0}}{2 \pi} \frac{1}{\widetilde{\rho}\left(k_{0}, k_{1}\right)}\left[\frac{1}{2} \frac{\partial^{2}}{\partial k_{0}^{2}} \widetilde{\rho}\left(k_{0}, k_{1}\right)-1\right] \\
r_{2}(L)=\frac{1}{L}\left[\sum_{l=1}^{L} \int \frac{d k_{0}}{2 \pi} \frac{1}{\widetilde{\rho}\left(k_{0}, k_{1}\right)}-\int \frac{d k_{0}}{2 \pi} \frac{1}{\hat{k}_{0}^{2}}\right]
\end{gathered}
$$

where the different dependence on the (continuous) time component $k_{0}$ and the (discrete) space components

$$
k_{1}=\frac{2 \pi l}{L}, \quad l=1, \ldots L
$$

is made explicit, and 


$$
\hat{k}^{2}=4 \sin ^{2}(k / 2)
$$

is the standard action momentum space representation of the inverse propagator. Moreover we obtained

$$
\begin{gathered}
s_{1}(L)=-\frac{1}{L} \sum_{l=1}^{L} \int \frac{d k_{0}}{2 \pi} \frac{8}{\widetilde{\rho}\left(k_{0}, k_{1}\right)}\left[\frac{\partial}{\partial q_{0}} \frac{\partial}{\partial q_{0}^{\prime}} c\left(q_{0}, 0 ; q_{0}^{\prime}, 0 ; k_{0}, k_{1}\right)\right]_{q_{0}=q_{0}^{\prime}=k_{0} / 2}, \\
s_{2}(L)=\frac{2}{L} \sum_{l=1}^{L} \int \frac{d k_{0}}{2 \pi} \frac{1}{\widetilde{\rho}\left(k_{0}, k_{1}\right)}\left[\frac{\partial^{2}}{\partial q_{0}^{2}} c\left(q_{0}, 0 ; 0 ; 0\right)-\frac{\partial^{2}}{\partial q_{0}^{2}} c\left(q_{0}, 0 ; k_{0}, k_{1} ; 0\right)\right]_{q_{0}=0} .
\end{gathered}
$$

Since the low-momentum behavior of $\widetilde{\rho}$ is dictated by the continuum limit to be

$$
\widetilde{\rho}\left(k_{0}, k_{1}\right) \longrightarrow k_{0}^{2}+k_{1}^{2}+O\left(k^{4}\right)
$$

and the low-momentum behavior of $\widetilde{c}$ is assumed to be a regular function of $k$, in the large $L$ limit the only singular dependence on $L$ is expected to be originated from $r_{2}(L)$. This singularity is simply parametrized by

$$
r_{2}(L)=\frac{1}{2 \pi} \ln \frac{L}{a}+\text { regular terms, }
$$

as predicted by our renormalization group considerations.

We tested our expressions of $r_{i}(L), s_{i}(L)$ against explicit (finite $L$, finite $T \gg L$ ) evaluation of the one-loop contribution to $m(L) L$ (i.e. using Eq. (11)). The propagator $P_{x y}$ wad found by numerically solving Eq. (10). We found full agreement for various forms of the action, including Symanzik off-shell [10] and on-shell [11] improved versions and mixed $O(N)-R P^{N-1}$ models. The correct large- $N$ limit is also reproduced. Furthermore the $L \rightarrow \infty$ limits of $r_{i}, s_{i}$ may be shown to agree in general with the computation of Ref. [3] of the $\Lambda$-parameter ratios.

Our expressions allow a rather direct testing of the effects that an arbitrary choice of the action may have on the finite-space-volume, finite-size-scaling-violation effects at the one-loop level. They are especially useful in order to test the effects of a systematic treelevel Symanzik improvement. To this purpose we note that within Symanzik's program the quadrilinear couplings $c_{n_{1} n_{2} n_{3} n_{4}}$ are absent, and a systematic tree-level improvement can be achieved by choosing

$$
\widetilde{\rho}\left(k_{0}, k_{1}\right)=\sigma_{n}\left(k_{0}\right)+\sigma_{n}\left(k_{1}\right),
$$

where the index $n$ is related to the tree-level Symanzik's improvement degree. The family of inverse one-dimensional propagators $\sigma_{n}(k)$ is defined by

$$
\sigma_{n}(k)=\sum_{t=1}^{n} \frac{2}{t^{2}} \frac{(t !)^{2}}{(2 t) !}\left(\hat{k}^{2}\right)^{t}
$$

enjoying the basic property 


$$
\frac{1}{2} \frac{\partial^{2} \sigma_{n}(k)}{\partial k^{2}}=1-\frac{(n !)^{2}}{(2 n) !}\left(\hat{k}^{2}\right)^{n}
$$

For $n=1$ we recover the standard action.

By substituting the above relationships into the expression of of $r_{i}(L)$ (cfr. Eqs. (16) and (17)) one finds that, for $L$ sufficiently larger than $n$,

$$
\begin{gathered}
r_{1}^{(n)}(L)=\bar{r}_{1}^{(n)}+O\left[\left(a^{2} / L^{2}\right)^{n}\right], \\
r_{2}^{(n)}(L)=\frac{1}{2 \pi} \ln \frac{L}{a}+\bar{r}_{2}^{(n)}+O\left[\left(a^{2} / L^{2}\right)^{n}\right],
\end{gathered}
$$

where $\bar{r}_{i}^{(n)}$ are constants that can be computed in the infinite space-volume limit. Corrections are power-series expandable in the powers of $a^{2} / L^{2}$. The numerically evaluated coefficients of the leading-order power correction turn out to rapidly grow with increasing $n$, suggesting some non-uniformity in the large- $n$ limit.

The above results exhibit explicitly the correct continuum RG one-loop coefficient, the cancellation of all dependence on $\ln (L / a)$ in finite lattice effects and the cancellation of all $\left(a^{2} / L^{2}\right)^{t}$ dependence for $t<n$ in the $n$-th order tree-improved action. This is exactly the pattern described in our general analysis. The action defined by the (formal) limit $n \rightarrow \infty$ is what we call the "Symanzik tree perfect" action. It essentially amounts to a "SLAC derivative" lattice version of the continuum action, and shows no power-like corrections to scaling when the limit $L \rightarrow \infty$ is taken before the limit $n \rightarrow \infty$. One must not however forget that, since this action involves infinite-range correlations, at finite $L$ there are finite-volume effects related to the unavoidable truncation. These effects appear as $O\left(1 / L^{2}\right)$ corrections when $n \gg L$. We insist that the relevance of the result is purely conceptual, in that it shows that tree-improvement may lead in this case to cancellation of one-loop finite-volume effects.

The "Symanzik tree-perfect" action allows exact evaluation of some $n \rightarrow \infty$ limits, that are obtained by setting $\sigma_{\infty}(k)=k^{2}$ and integrating between $-\pi$ and $\pi$ in the $k_{0}, k_{1}$ variables. We obtained the following analytical results:

$$
\begin{aligned}
\bar{r}_{1}^{(\infty)} & =\frac{1}{4 \pi}, \\
\bar{r}_{2}^{(\infty)} & =\frac{1}{2 \pi}\left(\gamma_{E}-\ln 2-\frac{2}{\pi} G\right),
\end{aligned}
$$

where $G$ is Catalan's constant. The $n \rightarrow \infty$ limit is reached with $O\left(n^{-1 / 2}\right)$ corrections in the case of $\bar{r}_{1}$, and $O\left(n^{-1}\right)$ corrections in the case of $\bar{r}_{2}$. For comparison we recall that for the stardard nearest-neighbour action [5]:

$$
\begin{aligned}
& \bar{r}_{1}^{(1)}=\frac{1}{4}, \\
& \bar{r}_{2}^{(1)}=\frac{1}{2 \pi}\left(\gamma_{E}+\frac{1}{2} \ln 2-\ln \pi\right) .
\end{aligned}
$$

Because of the recent upsurge of interest in the so-called "on-shell improved" lattice actions, we also considered the simplest representative of this class of actions in the context 
of two-dimensional $O(N) \sigma$ models. Limiting ourselves to $O\left(a^{2}\right)$ tree improvement we choose $c_{n_{1} n_{2} n_{3} n_{4}}=0$ and the inverse propagator

$$
\widetilde{\rho}_{\text {os }}\left(k_{0}, k_{1}\right)=\hat{k}_{0}^{2}+\hat{k}_{1}^{2}-\frac{1}{6} \hat{k}_{0}^{2} \hat{k}_{1}^{2}=k_{0}^{2}+k_{1}^{2}-\frac{1}{12}\left(k_{0}^{2}+k_{1}^{2}\right)^{2}+O\left(k^{6}\right) .
$$

Performing the integrations in the variable $k_{0}$ we found

$$
\begin{gathered}
r_{1}(L)=\frac{1}{2}-\frac{1}{3 L} \sum_{l=1}^{L} \frac{\hat{k}_{1}^{2}}{2 \sqrt{\hat{k}_{1}^{2}+\frac{1}{12} \hat{k}_{1}^{4}}}=0.30755 \ldots+O\left(\frac{a^{2}}{L^{2}}\right) \\
r_{2}(L)=\frac{1}{L} \sum_{l=1}^{L-1} \frac{1}{2 \sqrt{\hat{k}_{1}^{2}+\frac{1}{12} \hat{k}_{1}^{4}}}=\frac{1}{2 \pi} \ln \frac{L}{a}-0.0028979 \ldots+O\left(\frac{a^{4}}{L^{4}}\right)
\end{gathered}
$$

Hence only for large $N$ on-shell improvement is effective in reducing the scaling violations in the finite-space-volume mass-gap, even if this is an asymptotic (spectral) property of the model. $r_{1}(L)$ does not behave better than the corresponding quantity calculated with the standard action.

A similar pattern we found when considering the action involving only the quadratic terms of the "Hasenfratz-Niedermayer" perfect action. This is not unexpected when we consider the low-momentum expansion of the corresponding function $\widetilde{\rho}[12]$ and realize that it is consistent with $O\left(a^{2}\right)$ on-shell improvement. In Ref. [1] it was verified that the inclusion of the "perfect" quartic couplings in the action has the effect of removing all power-law dependence on lattice artifacts. Our results for $r_{i}$ and $s_{i}$ should now allow to establish the more general conditions under which, starting from a given form of the bilinear interaction $\rho_{x y}$ (dictated by the specific RG transformations considered), one may choose functions $\widetilde{c}$ such that all non-scaling one-loop finite lattice effects are removed, at least in the finitespace-volume mass gap.

We conclude with a few remarks about four-dimensional lattice QCD. Similarly to the finite-volume mass gap in 2-d $O(N) \sigma$ models, one may define observables which do not present $O\left(a^{k} \ln a\right)$ terms in their one-loop perturbative evaluation even when using the standard Wilson action. In the following we give an example of such a quantity. Consider the correlation of two Polyakov lines $P(\vec{r})$ (or equivalently Wilson loops $T \times R$ with $T \gg R$ )

$$
C(\vec{r})=\frac{1}{N}\langle\operatorname{Tr} P(\vec{r}) P(0)\rangle .
$$

The perturbative expansion of $C(\vec{r})$ has been considered in Ref. [7] within a study of the Symanzik's program in lattice gauge theories. Using the Wilson action and for an infinite lattice

$$
C(\vec{r})=1+T c_{F} g^{2} \int \frac{d^{3} k}{(2 \pi)^{3}} \frac{e^{i \vec{k} \cdot \vec{r}}-1}{\hat{k}_{1}^{2}+\hat{k}_{2}^{2}+\hat{k}_{3}^{2}}+O\left(g^{4}\right),
$$

where $T$ is the length of the Polyakov line. As shown in Ref. [0], the one-loop contribution to 


$$
A(\vec{r}) \equiv \frac{1}{T} \ln C(\vec{r})
$$

contains $O\left(g^{4} a^{2 k} \ln a\right)$ terms. This is not unexpected because at tree level $O\left(a^{2}\right)$ finite lattice spacing corrections are present.

Let us now introduce the wall-wall correlation

$$
W(z) \equiv \frac{1}{L_{x} L_{y}} \sum_{x y} C(\vec{r}) .
$$

It is easy to prove that

$$
D(z) \equiv \frac{1}{c_{F} T} \ln \frac{W(z+1)}{W(z)}=g^{2}+O\left(g^{4}\right)
$$

similarly to $m(L) L$ in two-dimensional $O(N) \sigma$ models. Then, unlike $A(\vec{r})$, the $O\left(g^{4}\right)$ corrections in $D(z)$ will not contain $O\left(g^{4} a^{2 k} \ln a\right)$ terms, and its perturbative expansion looks like that of $m(L) L$ with the substitution $z \rightarrow L$. Notice that the continuum limit of $D(z)$ defines a running coupling constant obeying a homogeneous renormalization group equation with a corresponding beta-function.

\section{ACKNOWLEDGMENTS}

It is a pleasure to thank Andrea Pelissetto for useful and stimulating discussions. 


\section{REFERENCES}

[1] K. Wilson and J. Kogut, Phys. Rep. C12 (1974) 75; K. Wilson, Rev. Mod. Phys. 47 (1975) 773, $5 \mathbf{5}$ (1983) 583.

[2] K. Symanzik, in "Mathematical problems in theoretical physics", eds. R. Schrader et al. (Springer, Berlin, 1982), Lecture Notes in Physics 153; Nucl. Phys. B226 (1983) 187, 205.

[3] P. Hasenfratz and F. Niedermayer, Nucl. Phys. B414 (1994) 785.

[4] F. Farchioni, P. Hasenfratz, F. Niedermayer, and A. Papa, Nucl. Phys. B454 (1995) 638.

[5] M. Lüscher, Phys. Lett. 118B (1982) 391; unpublished notes.

[6] M. Lüscher, P. Weisz, and U. Wolff, Nucl. Phys. B359 (1991) 221.

[7] G. Curci, P. Menotti, and G. Paffuti, Phys. Lett. 130B (1983) 205.

[8] J. M. Cline, Phys. Lett. 173B (1986) 173.

[9] P. Hasenfratz, Phys. Lett. 141B (1984) 385.

[10] G. Martinelli, G. Parisi, R. Petronzio, Phys. Lett. 114B (1982) 251.

[11] M. Lüscher and P. Weisz, Comm. Math. Phys. 97 (1985) 159.

[12] T. DeGrand, A. Hasenfratz, P. Hasenfratz and F. Niedermayer, Nucl. Phys. B454 (1994) 587. 